\title{
Preoperative Hyponatremia And Hypocalcemia Predict Poor Prognosis In Elderly Gastric Cancer Patients
}

\author{
Jingxuan Xu (D)* \\ Xiaodong Chen* \\ Xiang Wang \\ Ce Zhu \\ Yuanbo $\mathrm{Hu}$ \\ Xinxin Yang \\ Chongyong $\mathrm{Xu}$ \\ Xian Shen
}

Department of Gastrointestinal Surgery, The Second Affiliated Hospital, Wenzhou Medical University, Wenzhou, Zhejiang,

People's Republic of China

*These authors contributed equally to this work
This article was published in the following Dove Press journal: Cancer Management and Research

Background: Hyponatremia and hypocalcemia are common in patients with cancer, but their prognostic impact in patients who underwent gastric cancer (GC) surgery has not been investigated. Therefore, this study aimed to determine the postoperative prognostic impact of hyponatremia and hypocalcemia in patients undergoing curative gastrectomy by age group. Materials and methods: GC patients preoperatively diagnosed with hyponatremia or hypocalcemia who underwent elective radical gastrectomy were retrospectively evaluated. The patients were divided into the elderly group ( $\geq 60$ years) and the young group ( $<60$ years), and then further based on their sodium and calcium levels. The effect of preoperative hyponatremia or hypocalcemia on postoperative complications (PCs) by age was determined using univariate and multivariate analyses. Overall survival (OS) was compared between the two groups using log rank test and Cox proportional hazards regression.

Results: Of the 842 patients evaluated, 36 (4.3\%) were categorized into the younger hyponatremia group; 64 (7.6\%), the elderly hyponatremia group; 48 (5.7\%), the young hypocalcemia group; and $128(15.2 \%)$, the elderly hypocalcemia group. Hyponatremia $(\mathrm{P}=0.001)$ and hypocalcemia $(\mathrm{P}=0.038)$ were independent risk factors for PCs in the elderly group. Further, hypocalcemia (hazard ratio (HR), 0.676; $\mathrm{P}=0.037$ ) was independently associated with shorter OS.

Conclusion: Preoperative hyponatremia and hypocalcemia predict poor outcomes in the elderly, but not in young GC patients. Further, hyponatremia and hypocalcemia in elderly GC patients should be corrected in the earliest time possible to obtain better clinical outcomes. Keywords: gastric cancer, hyponatremia, hypocalcemia, elderly patients, postoperative complications, overall survival

\section{Introduction}

Gastric cancer (GC) is an important cancer worldwide as it is the fifth most frequently diagnosed cancer, with more than 1,000,000 new cases diagnosed in 2018. ${ }^{1}$ Moreover, GC is one of the most common malignancies in China. ${ }^{2}$ With the rapid aging of the global population, the number of elderly patients with locally advanced GC is also increasing in China, with GC being the most common malignancy in the Chinese elderly population. ${ }^{3}$ Gastrectomy with precise lymph node dissection remains the main treatment modality for $\mathrm{GC},{ }^{4}$ but studies have shown a high incidence of postoperative complications (PCs) in the elderly at up to $18-32 \% .^{5}$ PCs are the primary cause of severe economic and social burden among elderly GC patients. Thus, timely intervention is crucial in elderly patients as this may decrease PCs and mortality. ${ }^{6}$
Correspondence: Chongyong Xu; Xian Shen

Department of Gastrointestinal Surgery, The Second Affiliated Hospital, Wenzhou Medical University, Wenzhou, Zhejiang Province, People's Republic of China Tel +86057788002709

Email stony693100@163.com; shenxian5166@gmail.com 
Hyponatremia and hypocalcemia are common in surgery, ${ }^{7}$ and it occurs in as high as $47 \%$ of hospitalized cancer patients. ${ }^{8}$ The prognostic significance of hyponatremia and hypocalcemia is well described for several tumor types, such as hypercalcemia for breast and kidney cancer and hyponatremia for small cell lung cancer, ${ }^{9}$ but has not been well studied in GC.

Serum sodium and calcium levels can be easily determined via routine plasma electrolyte tests. Hyponatremia (i.e., serum sodium level at lower limit of normal (LLN) $<135 \mathrm{mEq} / \mathrm{l})^{10}$ was considered to be an indicator of severity and prognosis of the underlying disease in various medical conditions, including liver cirrhosis ${ }^{11}$ and congestive heart failure. ${ }^{12}$ Further, hyponatremia can develop as a side effect of drug therapy. ${ }^{13}$ Meanwhile, hypocalcemia (i.e., serum calcium level $<$ LLN-8.0 mg/dL or $<$ LLN-2.0 mmol/L; ionized calcium $<$ LLN-1.0 mmol $/ \mathrm{L})^{10}$ often develops gradually and has nonspecific symptoms. Further, it may resemble the symptoms of the underlying malignancy and its treatment. $^{14}$

Hyponatremia and hypercalcemia are the most common cancer-related electrolyte disorders. However, the relationship between GC and hyponatremia or hypocalcemia remains unclear, and thus their management in cancer patients is yet to be established. Because elderly patients usually have poor oral intake, vomiting, diarrhea, and chronic blood loss, they may be more susceptible to hyponatremia or hypocalcemia. Thus, this study aimed to comprehensively evaluate the postoperative prognostic impact of hyponatremia or hypocalcemia in GC patients by age.

\section{Materials And Methods Study Design And Subjects}

This retrospective study was performed using data of GC patients who underwent radical gastrectomy at the Gastroenterology Department of the Second Affiliated Hospital of Wenzhou Medical University between June 2009 and February 2018.

The inclusion criteria were: i) gastric adenocarcinoma confirmed via preoperative gastroscopy and pathological examination and ii) scheduled for curative gastrectomy. The exclusion criteria were: i) radiological assessment in another hospital; ii) history of palliative resection; iii) history of emergency surgery for preoperative gastrointestinal bleeding; iv) history of immune system disease, uremia, and severe preoperative kidney function damage; v) and difference in pre- and postoperative diagnosis.
Data on 1) clinicodemographic characteristics including sex, age, electrolyte concentration, body mass index (BMI), preoperative comorbidities, Charlson score, albumin concentration, hemoglobin concentration, histological type, tumor location, tumor node-metastasis (TNM) stage, and tumor size; 2) operative characteristics including laparoscopic surgery (surgeries were mainly performed by laparoscopy except for reconstruction of the digestive tract), lymph node dissection, and type of resection and reconstruction; and 3) postoperative outcomes, including PCs and postoperative survival time were collected from medical records. The patients were divided into two groups by age at a cut-off of 60 years based on the report of the United Nations World Health Organization. ${ }^{15}$ Particularly, the young and the elderly group comprised those aged $<60$ years and $\geq 60$ years, respectively. Two experienced researchers graded PCs that occurred within 30 days after operation using the Clavien-Dindo classification. ${ }^{16,17}$ The study was approved by the Ethics Committee of the Second Affiliated Hospital of Wenzhou Medical University; all participants provided written informed consent before participating in the study.

\section{Diagnosis Of Electrolyte Abnormalities}

Hyponatremia and hypocalcemia were defined according to the Common Terminology Criteria of Adverse Events, version 4.03. ${ }^{10}$ Hyponatremia and hypocalcemia were acquired as a time-varying covariate because they could resolve and then recur. ${ }^{18}$ Therefore, the blood calcium and blood sodium concentrations determined after admission were used for the assessment of hyponatremia and hypocalcemia.

\section{Statistical Analysis}

A Kolmogorov-Smirnov test was conducted to evaluate the equality of constant parameter distribution. Normal distribution data are expressed as mean $\pm \mathrm{SD}$ deviations, while non-normal distribution data are expressed as mean and interquartile ranges (IQR). In univariate analysis, the independent $t$-test and Mann-Whitney $U$-test were used to analyze intergroup differences in continuous variables, and the chi-square test and Fisher's exact test were applied for categorical variables. Overall survival (OS) was defined as the time between the date of diagnosis and death or the date of last known follow-up. The Kaplan-Meier method and $\log$ rank test were used to estimate and compare survival based on specific factors. The Cox proportional hazard model was used to assess the risk ratio in univariate and multivariate analyses. All P-values were bilateral, and 
$\mathrm{P}<0.05$ was considered statistically significant. All statistical analyses were analyzed using SPSS software (version 23.0; SPSS Inc., Chicago, IL, USA).

\section{Results}

\section{Patient Characteristics}

Of the 869 patients initially identified, we excluded 27 patients whose serum calcium and sodium levels were not measured $(n=8)$, those who had metastases to other sites and underwent palliative surgery $(n=8)$, who were previously diagnosed with severe preoperative kidney function damage $(n=2)$, and whose postoperative diagnosis was different from the preoperative diagnosis $(n=9)$. Finally, the cohort comprised 842 patients who were accordingly grouped based on their sodium and calcium levels as the hyponatremia group $(\mathrm{n}=100(11.9 \%))$ and non-hyponatremia group $(\mathrm{n}=742$ $(88.1 \%))$, and the hypocalcemia group $(n=176(20.9 \%))$ and non-hypocalcemia group $(\mathrm{n}=666(79.1 \%))$.

The patient characteristics are shown in Table 1. Majority of patients were men $(n=627,74.5 \%)$, and the mean age in the overall cohort was $64.0(\mathrm{IQR}=56.0-72.0)$ years, indicating that majority were elderly. The median postoperative hospital stay was 16.1 (IQR=13.2-21.1) days, and the median hospitalization expense was $¥ 34,718.4$ ( IQR $=¥ 22,003.8-50,495.2$ ). The median preoperative hemoglobin and preoperative albumin levels were $124.0(\mathrm{IQR}=105.0-136.0) \mathrm{g} / \mathrm{l}$ and 36.1 $(\mathrm{IQR}=32.7-38.7) \mathrm{g} / \mathrm{l}$, respectively. The median postoperative survival time was 82.2 months (IQR=78.2-86.3) days.

\section{Correlation Between Clinicopathologic Characteristics And Hyponatremia}

BMI $(\mathrm{P}=0.048)$, tumor size $(\mathrm{P}=0.024)$, preoperative obstruction $(\mathrm{P}=0.003)$, and $\mathrm{PC}(\mathrm{P}=0.004)$ were significantly correlated with hyponatremia (Table 1). Meanwhile, there was no significant association between hyponatremia and sex, Charlson score, diabetes, T stage, hypertension, preoperative anemia, preoperative bleeding, or node status.

\section{Correlation between clinicopathologic characteristics and hypocalcemia}

Charlson score $(\mathrm{P}<0.001)$, tumor size $(\mathrm{P}<0.001)$, $\mathrm{T}$ stage $(\mathrm{P}=0.020)$, preoperative anemia $(\mathrm{P}<0.001)$, preoperative bleeding $(\mathrm{P}=0.003)$, preoperative obstruction $(\mathrm{P}<0.001)$, and $\mathrm{PC}(\mathrm{P}<0.001)$ were significantly correlated with hypocalcemia. Meanwhile, there was no significant association between hypocalcemia and sex, BMI, T stage, diabetes, hypertension, or node status.

\section{Univariate And Multivariate Analysis Of Factors Associated With Postoperative Complications}

Patients with hyponatremia or hypocalcemia had higher risk of PCs (Figure 1). Univariate analysis showed significant correlations between $\mathrm{PCs}$ and age $(\mathrm{P}<0.001)$, diabetes $(\mathrm{P}=0.037)$, Charlson score $(\mathrm{P}=0.003)$, hyponatremia $(\mathrm{P}=0.004)$, hypocalcemia $(\mathrm{P}<0.001)$, TNM stage $(\mathrm{P}=0.001)$, $\mathrm{T}$ stage $(\mathrm{P}=0.003)$, total gastrectomy $(\mathrm{P} \leq 0.001)$, open surgery $(\mathrm{P}=0.013)$, and reconstruction methods $(\mathrm{P}=0.001)$ (Table 2). Meanwhile, only age $(\mathrm{P}=0.008)$, hyponatremia $(\mathrm{P}=0.039)$, and hypocalcemia $(\mathrm{P}=0.023)$ remained to be independent risk factors for $\mathrm{PCs}$ in multivariate logistic regression analysis (Table 2).

\section{Univariate And Multivariate Analysis Of Factors Associated With Postoperative Complications In Young Patients}

The young and the elderly groups comprised 319 (37.9\%) and $523(82.1 \%)$ patients, respectively. In total, 52 (16.3\%) patients in the young group developed PCs. Univariate analysis showed significant correlations between PCs and Charlson score $(\mathrm{P}=0.001)$, pathologic type $(\mathrm{P}=0.033)$, and open surgery $(\mathrm{P}=0.026)$. Meanwhile, in multivariate logistic regression analysis, pathologic type was the only independent risk factor for PCs. Hyponatremia or hypocalcemia cannot predict PCs in young GC patients.

\section{Univariate And Multivariate Analysis Of Factors Associated With Postoperative Complications In Elderly Patients}

In the elderly group, 154 (29.4\%) patients developed PCs. In the univariate analysis, there were significant correlations between $\mathrm{PCs}$ and diabetes $(\mathrm{P}=0.050)$, hyponatremia ( $\mathrm{P}=0.001)$, hypocalcemia $(\mathrm{P}=0.003)$, TNM stage $(\mathrm{P}=0.018)$, anastomotic method $(\mathrm{P}=0.005)$, and total gastrectomy $(\mathrm{P}=0.001)$. In multivariate logistic regression analysis, there were significant correlations between $\mathrm{PCs}$ and diabetes $(\mathrm{P}=0.048)$, hyponatremia $(\mathrm{P}<0.001)$, and hypocalcemia $(\mathrm{P}=0.003)$ (Table 3).

\section{Survival Analysis Of Variables Associated With Hyponatremia Or Hypocalcemia In Young Patients}

As shown in Figure 1, GC patients with hypocalcemia had a poor outcome: the median survival time in patients with hypocalcemia was shorter than in those without 


\begin{tabular}{|c|c|c|c|c|c|c|c|c|c|c|c|}
\hline$a$ & & $\begin{array}{l}\text { 足 } \\
\text { مी } \\
0\end{array}$ & & & $\begin{array}{l}\stackrel{\tilde{m}}{\sigma} \\
\stackrel{0}{0}\end{array}$ & : & 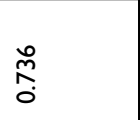 & $\stackrel{\infty}{\infty}$ & $\frac{\underline{n}}{\overline{0}}$ & $\begin{array}{l}\frac{*}{\circ} \\
\text { v } \\
\text { v }\end{array}$ & $\begin{array}{l}\frac{*}{\circ} \\
\text { o } \\
\text { v }\end{array}$ \\
\hline 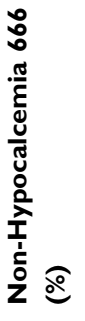 & 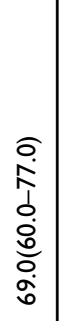 & 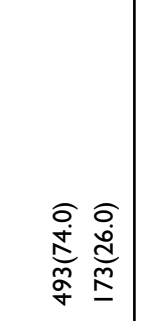 & 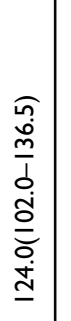 & 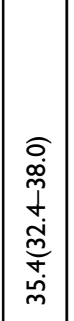 & 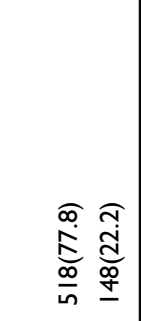 & 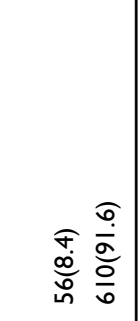 & 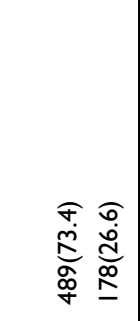 & 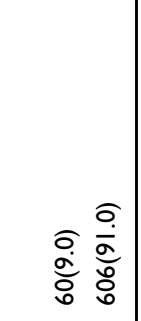 & 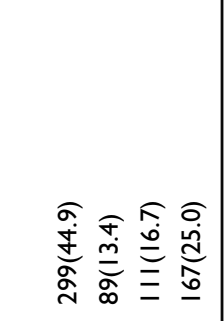 & 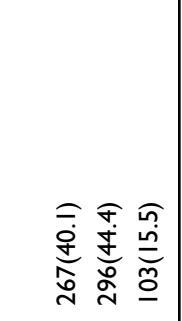 & 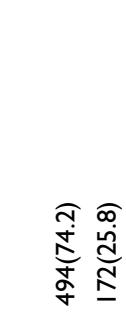 \\
\hline 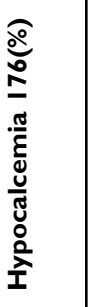 & 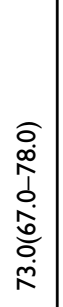 & 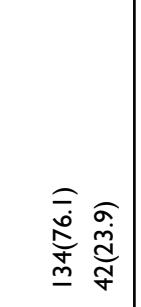 & 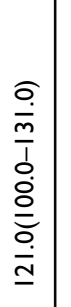 & 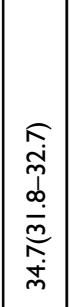 & 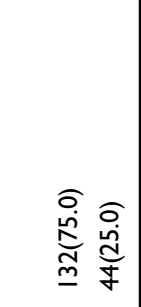 & 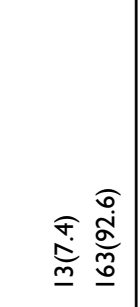 & 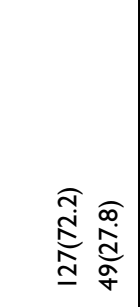 & 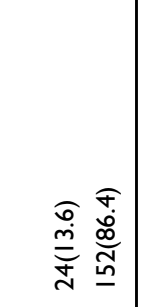 & 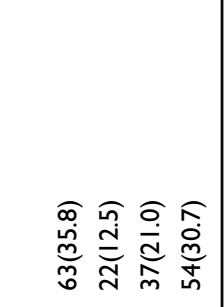 & 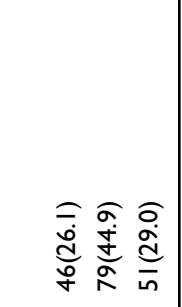 & 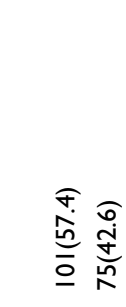 \\
\hline a & & $\frac{O}{0}$ & & & 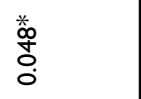 & 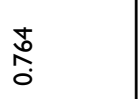 & $\begin{array}{l}\stackrel{\tilde{o}}{\sigma} \\
\hat{\sigma}\end{array}$ & $\begin{array}{l}\text { कo } \\
\stackrel{\text { t}}{0}\end{array}$ & : & $\frac{n}{\mathfrak{n}}$ & $\begin{array}{l}\text { 类 } \\
\text { O. } \\
0\end{array}$ \\
\hline 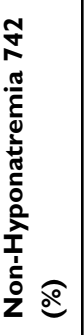 & 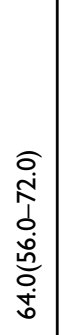 & 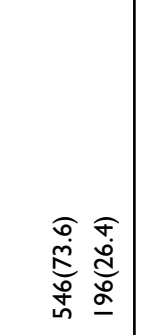 & 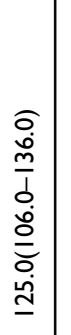 & $\left|\begin{array}{c}a \\
\infty \\
\infty \\
\tilde{o} \\
\tilde{j} \\
\tilde{n} \\
\tilde{n} \\
\tilde{m}\end{array}\right|$ & 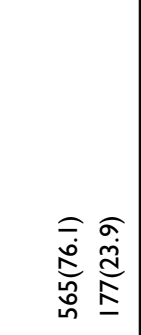 & 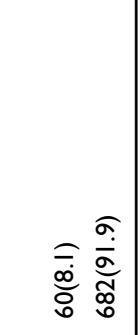 & 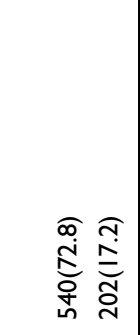 & 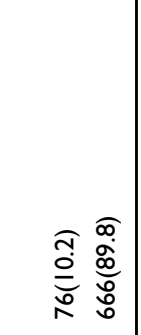 & 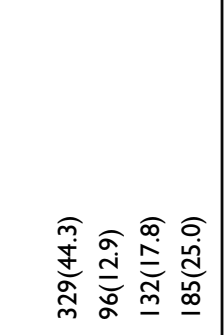 & 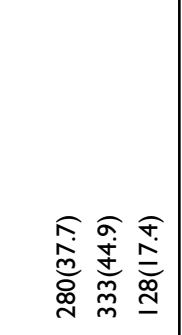 & 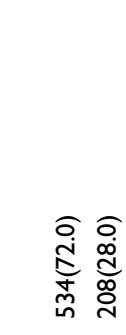 \\
\hline 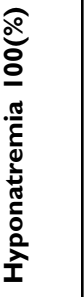 & 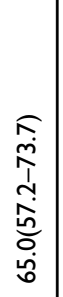 & 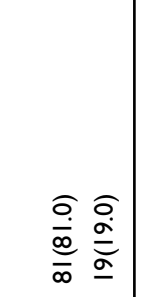 & 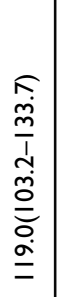 & 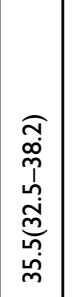 & 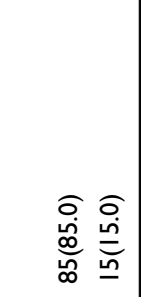 & 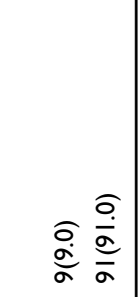 & 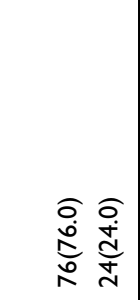 & 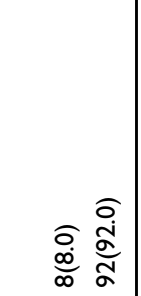 & 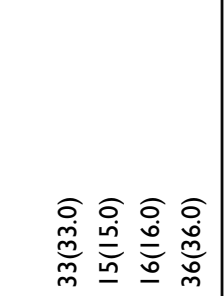 & 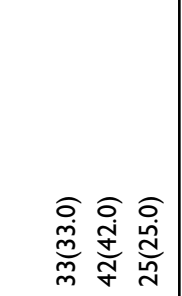 & $\begin{array}{ll}\frac{\partial}{0} & \frac{0}{\dot{0}} \\
\frac{0}{0} & \frac{\tilde{m}}{m}\end{array}$ \\
\hline 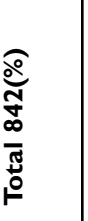 & 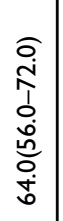 & 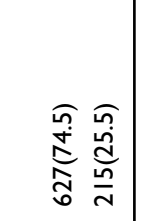 & 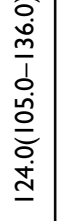 & 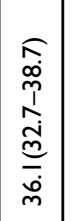 & 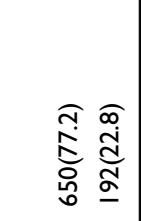 & 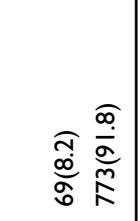 & 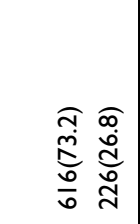 & 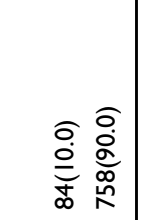 & 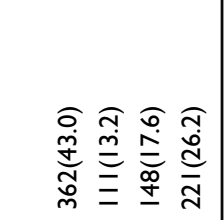 & 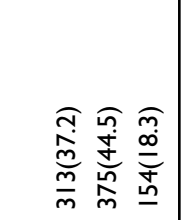 & 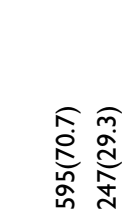 \\
\hline Lัّ & 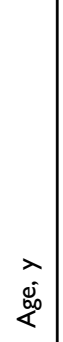 & 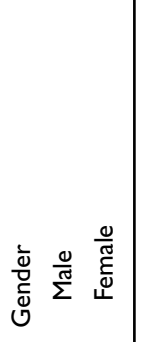 & 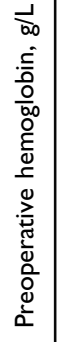 & 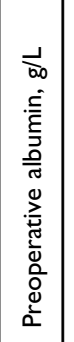 & 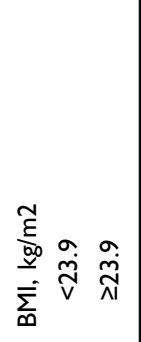 & 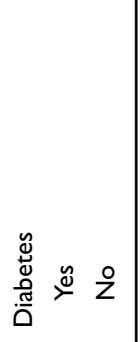 & 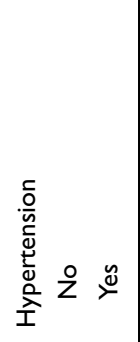 & 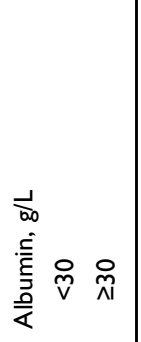 & 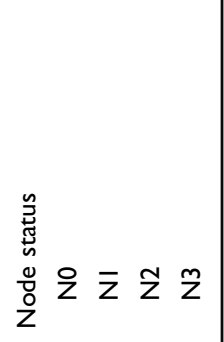 & 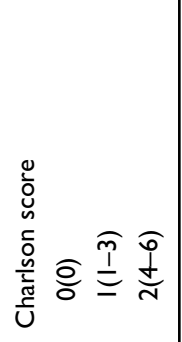 & 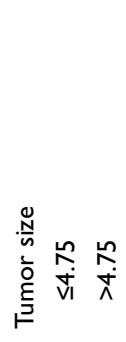 \\
\hline
\end{tabular}




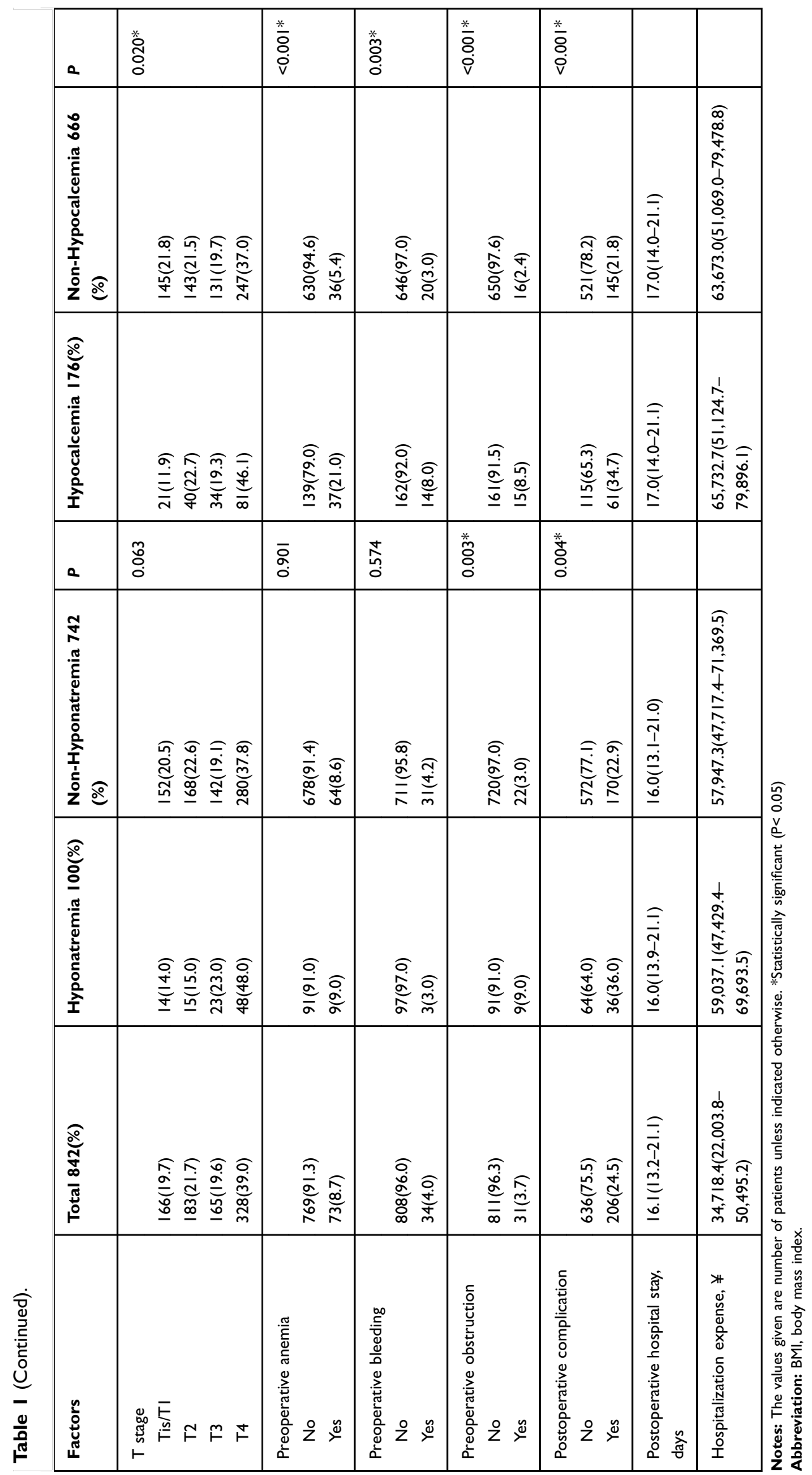



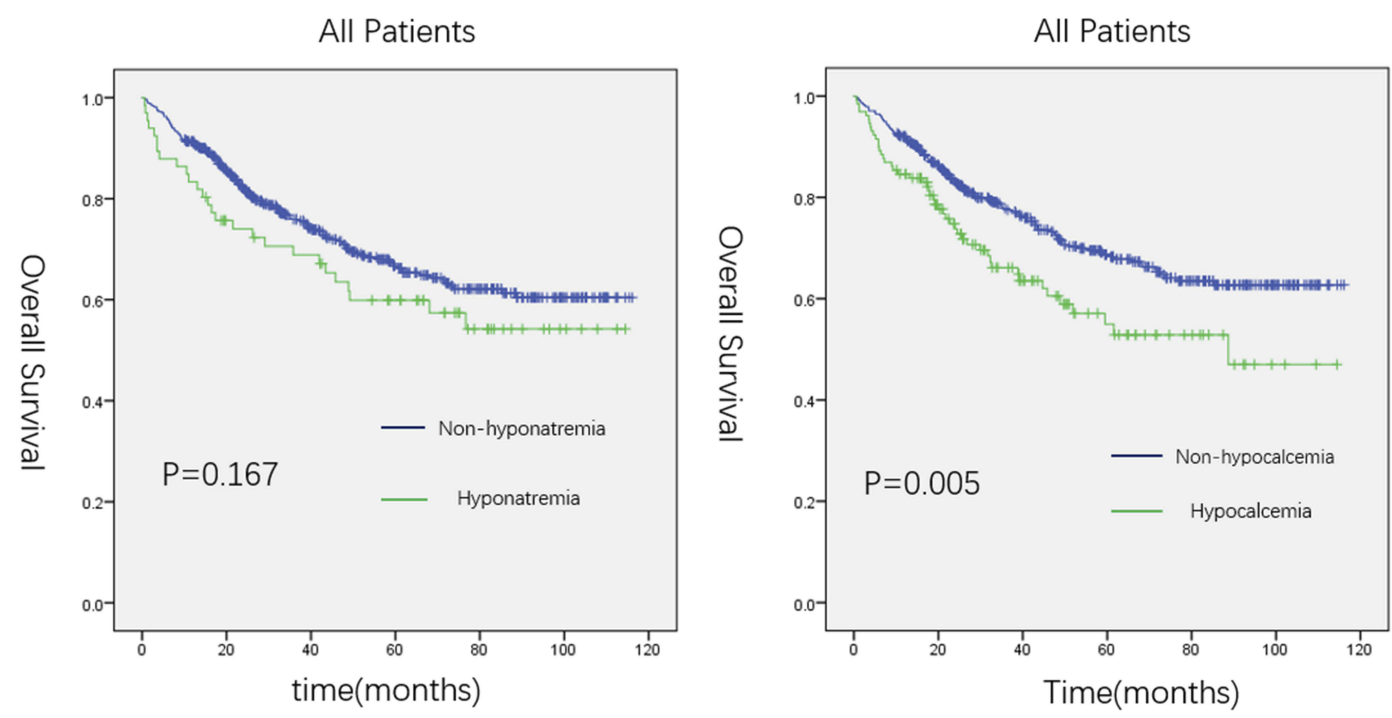

Figure I Kaplan-Meier curve for overall survival (OS) in patients with hyponatremia or hypocalcemia and those without.

hypocalcemia (70.7 months vs, 85.0 months, $\mathrm{P}=0.005)$. In the young group, univariate analysis showed that preoperative obstruction (HR, 4.300; 95\% CI, 1.310-14.119; $\mathrm{P}=0.016)$, readmission $(\mathrm{HR}, 3.438 ; 95 \% \mathrm{CI}, 1.052-$ 11.231; $\mathrm{P}=0.041)$, larger tumor size $(\mathrm{HR}, 4.245 ; 95 \% \mathrm{CI}$, 2.213-8.142; $\mathrm{P}<0.001)$, worse histopathological differentiation (HR, 0.424; 95\% CI, 0.209-0.862; $\mathrm{P}=0.018$ for differentiated type), higher TNM stage (HR, 19.029; 95\% CI, 2.341-154.706; $\mathrm{P}=0.006$ for stage II and HR, 33.578; 95\% CI, 4.572-246.582; $\mathrm{P}=0.001$ for stage III), total gastrectomy (HR, 0.418; 95\% CI, 0.209-0.832; $\mathrm{P}=0.013$ ), and anastomotic method (HR, 3.451; 95\% CI, 1.438-8.282; $\mathrm{P}=0.006$ for Billroth II and HR, 3.271; 95\% CI, 1.576$6.790 ; \mathrm{P}=0.001$ for Roux-en-y) were associated with poor OS. On multivariate analysis, readmission (HR, 3.150; 95\% CI, 1.638-6.056; $\mathrm{P}=0.001)$, higher TNM stage $(\mathrm{HR}$, 0.037; 95\% CI, 0.05-0.282; $\mathrm{P}=0.001$ for stage II), tumor location (HR, 0.052; 95\% CI, 0.004-0.748; $\mathrm{P}=0.030$ for gastric body and HR, 0.058; 95\% CI, 0.004-0.909; $\mathrm{P}=0.043$ for antrum), and anastomotic method (HR, 2.152; 95\% CI, 1.176-3.939; $\mathrm{P}=0.013$ for Roux-en-y) were independently associated with worse OS (Table 4). As shown in Figure 2A, both hyponatremia and hypocalcemia cannot predict the prognosis of young GC patients.

\section{Survival Analysis Of Variables Associated With Hyponatremia Or Hypocalcemia In Elderly Patients}

As shown in Figure 2B, Interestingly, hypocalcemia predicted poor outcome in the elderly patients: the median survival time in elderly patients with hypocalcemia was shorter than in those without hypocalcemia (55.3 months vs 77.9 months, $\mathrm{P}<0.001)$. In univariate analysis, hypocalcemia (HR, 1.876; 95\% CI, 1.358-2.592; $\mathrm{P}=0.001$ ) was associated with poor OS. Additionally, readmission (HR, 1.783; 95\% CI, 1.048-3.034; $\mathrm{P}=0.033$ ), larger tumor size (HR, 2.100; 95\% CI, 1.542-2.860; $\mathrm{P}<0.001$ ), worse histopathological differentiation (HR, 4.314; 95\% CI, 1.66011.209; $\mathrm{P}=0.003$ for undifferentiated type), higher TNM stage (HR, 2.486; 95\% CI, 1.311-4.713; $\mathrm{P}=0.005$ for stage II and HR, 5.087; 95\% CI, 3.064-8.446; $\mathrm{P}<0.001$ for stage III), total gastrectomy (HR, 0.554; 95\% CI, 0.397-0.775; $\mathrm{P}=0.001$ ), anastomotic method (HR, 2.889; 95\% CI, 1.918-4.353; $\mathrm{P}=0.001$ for Billroth II and HR, 2.266; 95\% CI, 1.594-3.221; $\mathrm{P}=0.001$ for Roux-en-y), grade $>2$ PC (HR, 1.623; 95\% CI, 1.176-2.240; $\mathrm{P}=0.003)$, multiple surgeries (HR, 1.568; 95\% CI, 1.139-2.159; $\mathrm{P}=0.006)$, and surgical method (HR, 0.068; 95\% CI, 0.010-0.489; $\mathrm{P}=0.008$ for laparoscopic) were associated with poor OS. No other clinicopathologic factors were statistically correlated to prognosis. On multivariate analysis, hypocalcemia (HR, 0.476; 95\% CI, 0.467-0.978; $\mathrm{P}=0.037$ ), worse histopathological differentiation (HR, 0.181; 95\% CI, 0.068$0.483 ; \mathrm{P}=0.001$ for differentiated type and $\mathrm{HR}, 0.277 ; 95 \%$ CI, 0.131-0.586; $\mathrm{P}=0.001$ for undifferentiated type), TNM stage (HR, 0.293; 95\% CI, 0.170-0.506; $\mathrm{P}=0.001$ for stage II and HR, 0.583; 95\% CI, 0.360-0.943; $\mathrm{P}<0.001$ for stage III), and surgical method (HR, 11.445; 95\% CI, 1.59082.356; $\mathrm{P}=0.015$ for laparoscopic) were independently associated with worse OS (Table 4). 
Table 2 Univariate Analysis And Multivariate Analysis Of The Risk Of Postoperative Complication

\begin{tabular}{|c|c|c|c|c|}
\hline \multirow[t]{2}{*}{ Factors } & \multirow[t]{2}{*}{ Non-PC Group 636(\%) } & \multirow[t]{2}{*}{ PC Group 206 (\%) } & \multirow{2}{*}{$\begin{array}{l}\text { Univariate Analysis } \\
P\end{array}$} & \multirow{2}{*}{$\begin{array}{l}\text { Multivariate Analysis } \\
P\end{array}$} \\
\hline & & & & \\
\hline Gender & & & 0.532 & \\
\hline Male & $477(75.0)$ & $150(72.8)$ & & \\
\hline Female & $159(25.0)$ & $56(17.2)$ & & \\
\hline Age, y & & & $<0.00 I^{*}$ & $0.008 *$ \\
\hline$<60$ & $262(4 I .2)$ & $57(27.7)$ & & \\
\hline$\geq 60$ & $374(58.8)$ & $149(72.3)$ & & \\
\hline $\mathrm{BMI}, \mathrm{kg} / \mathrm{m} 2$ & & & 0.337 & \\
\hline$<23.9$ & $496(78.0)$ & I54(74.8) & & \\
\hline$\geq 23.9$ & $140(22.0)$ & $52(25.2)$ & & \\
\hline Diabetes & & & $0.037^{*}$ & \\
\hline No & $591(92.9)$ & |82(88.3) & & \\
\hline Yes & $45(7.1)$ & $24(I I .7)$ & & \\
\hline Hypertension & & & 0.624 & \\
\hline No & $468(73.6)$ & |48(7|.8) & & \\
\hline Yes & $168(26.4)$ & $58(18.2)$ & & \\
\hline Preoperative anemia & & & 0.143 & \\
\hline No & $586(92.1)$ & I83(88.8) & & \\
\hline Yes & $50(7.9)$ & $23(I I .2)$ & & \\
\hline Preoperative bleeding & & & 0.493 & \\
\hline No & $612(96.2)$ & $196(95.1)$ & & \\
\hline Yes & $24(3.8)$ & $10(4.9)$ & & \\
\hline Preoperative obstruction & & & 0.860 & \\
\hline No & $613(96.4)$ & 198(96.I) & & \\
\hline Yes & $23(3.6)$ & $8(3.9)$ & & \\
\hline Charlson score & & & $0.003^{*}$ & \\
\hline $0(0)$ & $242(38.1)$ & $7 I(34.5)$ & & \\
\hline$I(I-3)$ & $294(46.2)$ & $81(39.3)$ & & \\
\hline $2(4-6)$ & $100(15.7)$ & $54(26.2)$ & & \\
\hline Histologic type & & & 0.091 & \\
\hline Signet-ring cell carcinoma & $8 I(12.7)$ & $17(8.3)$ & & \\
\hline Differentiated & $540(84.9)$ & I87(90.8) & & \\
\hline Undifferentiated & $15(2.4)$ & $2(0.9)$ & & \\
\hline Hyponatremia & & & $0.004 *$ & $0.039 *$ \\
\hline No & $572(89.9)$ & $170(82.5)$ & & \\
\hline Yes & $64(10.1)$ & $36(17.5)$ & & \\
\hline Hypocalcemia & & & $<0.00 I^{*}$ & $0.023 *$ \\
\hline No & $521(81.9)$ & $145(70.4)$ & & \\
\hline Yes & $115(18.1)$ & $61(29.6)$ & & \\
\hline Albumin, $g / L$ & & & 0.904 & \\
\hline$\geq 30$ & $573(90.1)$ & 185(89.8) & & \\
\hline$<30$ & $63(9.9)$ & $21(10.2)$ & & \\
\hline
\end{tabular}

(Continued) 
Table 2 (Continued).

\begin{tabular}{|c|c|c|c|c|}
\hline \multirow[t]{2}{*}{ Factors } & \multirow[t]{2}{*}{ Non-PC Group 636(\%) } & \multirow[t]{2}{*}{ PC Group 206 (\%) } & \multirow{2}{*}{$\begin{array}{l}\text { Univariate Analysis } \\
P\end{array}$} & \multirow{2}{*}{$\begin{array}{l}\text { Multivariate Analysis } \\
P\end{array}$} \\
\hline & & & & \\
\hline $\begin{array}{l}\text { Hemoglobin, g/L } \\
\quad \geq 100 \\
\quad<100\end{array}$ & $\begin{array}{l}510(80.2) \\
126(19.8)\end{array}$ & $\begin{array}{l}160(77.7) \\
46(22.3)\end{array}$ & 0.436 & \\
\hline $\begin{array}{c}\text { TNM } \\
\text { 0/I } \\
\text { II } \\
\text { III }\end{array}$ & $\begin{array}{l}218(34.3) \\
103(17.0) \\
315(48.7)\end{array}$ & $\begin{array}{l}49(23.8) \\
25(12.1) \\
132(64.1)\end{array}$ & $0.001 *$ & \\
\hline $\begin{array}{l}\text { Node status } \\
\text { N0 } \\
\text { NI } \\
\text { N2 } \\
\text { N3 }\end{array}$ & $\begin{array}{l}290(45.6) \\
81(12.7) \\
108(17.0) \\
157(24.7)\end{array}$ & $\begin{array}{l}72(35.0) \\
30(14.6) \\
40(19.4) \\
64(31.1)\end{array}$ & 0.059 & \\
\hline $\begin{array}{l}\text { T stage } \\
\text { TI } \\
\text { T2 } \\
\text { T3 } \\
\text { T4 }\end{array}$ & $\begin{array}{l}|4|(22.2) \\
|4|(22.2) \\
\mid 25(\mid 9.7) \\
229(35.9)\end{array}$ & $\begin{array}{l}25(12.1) \\
42(20.4) \\
40(19.4) \\
99(48.1)\end{array}$ & $0.003 *$ & \\
\hline $\begin{array}{l}\text { Tumor location } \\
\text { Cardia } \\
\text { Corpus } \\
\text { Pylorus } \\
\text { Total }\end{array}$ & $\begin{array}{l}|| 2(\mid 7.6) \\
|4|(22.2) \\
380(59.7) \\
3(0.5)\end{array}$ & $\begin{array}{l}43(20.9) \\
52(25.2) \\
108(52.4) \\
3(1.5)\end{array}$ & 0.165 & \\
\hline $\begin{array}{l}\text { Tumor size } \\
\quad \leq 4.75 \\
>4.75\end{array}$ & $\begin{array}{l}459(72.2) \\
177(17.8)\end{array}$ & $\begin{array}{l}136(66.0) \\
70(34.0)\end{array}$ & 0.092 & \\
\hline $\begin{array}{l}\text { Pathological type } \\
\text { Non-ulcerative } \\
\text { Ulcerative }\end{array}$ & $\begin{array}{l}509(80.0) \\
127(20.0)\end{array}$ & $\begin{array}{l}168(81.6) \\
38(18.4)\end{array}$ & 0.632 & \\
\hline $\begin{array}{l}\text { Total gastrectomy } \\
\text { No } \\
\text { Yes }\end{array}$ & $\begin{array}{l}515(81.0) \\
121(19.0)\end{array}$ & $\begin{array}{l}142(68.9) \\
64(31.1)\end{array}$ & $<0.00 I^{*}$ & \\
\hline $\begin{array}{l}\text { Surgical method } \\
\text { Open } \\
\text { Laparoscopic }\end{array}$ & $\begin{array}{l}560(88.1) \\
76(11.9)\end{array}$ & $\begin{array}{l}194(94.2) \\
12(15.8)\end{array}$ & $0.013^{*}$ & \\
\hline $\begin{array}{l}\text { Combined operation } \\
\text { No } \\
\text { Yes }\end{array}$ & $\begin{array}{l}581(91.4) \\
55(8.6)\end{array}$ & $\begin{array}{l}179(87.0) \\
27(13.0)\end{array}$ & 0.061 & \\
\hline $\begin{array}{l}\text { Reconstruction methods } \\
\text { Billroth I } \\
\text { Billroth II } \\
\text { Roux-en-Y }\end{array}$ & $\begin{array}{l}370(58.2) \\
108(17.0) \\
158(24.8)\end{array}$ & $\begin{array}{l}93(45.1) \\
35(17.0) \\
78(37.9)\end{array}$ & $0.001 *$ & \\
\hline
\end{tabular}

Note: *Statistically significant $(P<0.05)$.

Abbreviation: PC, postoperative complication. 
Table 3 Univariate Analysis And Multivariate Analysis Of The Risk Of Postoperative Complication In Young Patients And Elderly Patients

\begin{tabular}{|c|c|c|c|c|c|c|c|c|}
\hline \multirow[t]{2}{*}{ Factors } & \multicolumn{4}{|c|}{ Young Patients $(<60 y)$} & \multicolumn{4}{|c|}{ Elderly patients $(\geq 60 y)$} \\
\hline & $\begin{array}{l}\text { Non-PC } \\
\text { Group } 242 \\
\text { (\%) }\end{array}$ & $\begin{array}{l}\text { PC } \\
\text { Group } \\
\text { 52(\%) }\end{array}$ & $\begin{array}{l}\text { Univariate } \\
\mathbf{p}\end{array}$ & $\begin{array}{l}\text { Multivariate } \\
\text { P }\end{array}$ & $\begin{array}{l}\text { Non-PC } \\
\text { Group } 394 \\
\text { (\%) }\end{array}$ & $\begin{array}{l}\text { PC } \\
\text { Group } \\
\text { I54(\%) }\end{array}$ & $\begin{array}{l}\text { Univariate } \\
\mathbf{p}\end{array}$ & $\begin{array}{l}\text { Multivariate } \\
\text { p }\end{array}$ \\
\hline $\begin{array}{l}\text { Gender } \\
\text { Male } \\
\text { Female }\end{array}$ & $\begin{array}{l}165(68.2) \\
77(31.8)\end{array}$ & $\begin{array}{l}36(69.2) \\
16(30.8)\end{array}$ & 0.883 & & $\begin{array}{l}312(79.2) \\
82(20.8)\end{array}$ & $\begin{array}{l}\text { II } 4(74.0) \\
40(26.0)\end{array}$ & 0.192 & \\
\hline $\begin{array}{l}\text { BMI }(\mathrm{kg} / \mathrm{m} 2) \\
\quad<23.9 \\
\geq 23.9\end{array}$ & $\begin{array}{l}194(80.2) \\
48(19.8)\end{array}$ & $\begin{array}{l}36(69.2) \\
16(30.8)\end{array}$ & 0.083 & & $\begin{array}{l}302(76.6) \\
92(23.4)\end{array}$ & $\begin{array}{l}118(76.6) \\
36(23.4)\end{array}$ & 0.995 & \\
\hline $\begin{array}{l}\text { Charison score } \\
\qquad \begin{array}{l}0(0) \\
I(I-3) \\
2(4-6)\end{array}\end{array}$ & $\begin{array}{l}122(50.4) \\
98(40.5) \\
22(9.1)\end{array}$ & $\begin{array}{l}31(59.6) \\
9(17.3) \\
12(23.1)\end{array}$ & $0.001 *$ & & $\begin{array}{l}120(30.5) \\
196(49.7) \\
78(19.8)\end{array}$ & $\begin{array}{l}40(26.0) \\
72(46.8) \\
42(27.2)\end{array}$ & 0.150 & \\
\hline $\begin{array}{l}\text { Preoperative anemia } \\
\text { No } \\
\text { Yes }\end{array}$ & $\begin{array}{l}229(94.6) \\
13(5.4)\end{array}$ & $\begin{array}{l}47(90.4) \\
5(9.6)\end{array}$ & 0.247 & & $\begin{array}{l}357(90.6) \\
37(9.4)\end{array}$ & $\begin{array}{l}136(88.3) \\
18(11.7)\end{array}$ & 0.421 & \\
\hline $\begin{array}{l}\text { Preoperative bleeding } \\
\text { No } \\
\text { Yes }\end{array}$ & $\begin{array}{l}234(96.7) \\
8(3.3)\end{array}$ & $\begin{array}{l}49(94.2) \\
3(5.8)\end{array}$ & 0.396 & & $\begin{array}{l}378(95.9) \\
16(4.1)\end{array}$ & $\begin{array}{l}147(95.5) \\
7(4.5)\end{array}$ & 0.799 & \\
\hline $\begin{array}{l}\text { Preoperative obstruction } \\
\text { No } \\
\text { Yes }\end{array}$ & $\begin{array}{l}234(96.7) \\
8(3.3)\end{array}$ & $\begin{array}{l}5 \mathrm{I}(98.1) \\
\mathrm{I}(\mathrm{I} .9)\end{array}$ & 0.599 & & $\begin{array}{l}379(96.2) \\
15(3.8)\end{array}$ & $\begin{array}{l}\text { I47(95.5) } \\
7(4.5)\end{array}$ & 0.692 & \\
\hline $\begin{array}{l}\text { Hypertension } \\
\text { No } \\
\text { Yes }\end{array}$ & $\begin{array}{l}208(86.0) \\
34(14.0)\end{array}$ & $\begin{array}{l}44(84.6) \\
8(15.4)\end{array}$ & 0.803 & & $\begin{array}{l}260(66.0) \\
134(34.0)\end{array}$ & $\begin{array}{l}104(67.5) \\
50(32.5)\end{array}$ & 0.731 & \\
\hline $\begin{array}{l}\text { Diabetes } \\
\text { No } \\
\text { Yes }\end{array}$ & $\begin{array}{l}229(94.6) \\
13(5.4)\end{array}$ & $\begin{array}{l}49(94.2) \\
3(5.8)\end{array}$ & 0.909 & & $\begin{array}{l}362(91.9) \\
32(8.1)\end{array}$ & $\begin{array}{l}133(86.4) \\
21(13.6)\end{array}$ & $0.050 *$ & $0.048^{*}$ \\
\hline $\begin{array}{l}\text { Hyponatremia } \\
\text { No } \\
\text { Yes }\end{array}$ & $\begin{array}{l}215(88.8) \\
27(11.2)\end{array}$ & $\begin{array}{l}48(92.3) \\
4(7.7)\end{array}$ & 0.460 & & $\begin{array}{l}357(90.6) \\
37(9.4)\end{array}$ & $\begin{array}{l}122(79.2) \\
32(20.8)\end{array}$ & $0.001 *$ & $0.001 *$ \\
\hline $\begin{array}{l}\text { Hypocalcemia } \\
\text { No } \\
\text { Yes }\end{array}$ & $\begin{array}{l}209(86.4) \\
33(13.6)\end{array}$ & $\begin{array}{l}42(80.8) \\
10(19.2)\end{array}$ & 0.300 & & $\begin{array}{l}312(79.2) \\
82(20.8)\end{array}$ & $\begin{array}{l}103(66.9) \\
51(33.1)\end{array}$ & 0.003 & $0.038^{*}$ \\
\hline $\begin{array}{l}\text { Albumin, g/L } \\
\quad \geq 100 \\
\quad<100\end{array}$ & $\begin{array}{l}225(93.0) \\
17(7.0)\end{array}$ & $\begin{array}{l}49(94.2) \\
3(5.8)\end{array}$ & 0.744 & & $\begin{array}{l}348(88.3) \\
46(11.7)\end{array}$ & $\begin{array}{l}\mid 36(88.3) \\
\mid 8(\mid 1.7)\end{array}$ & 0.997 & \\
\hline $\begin{array}{l}\text { Hemoglobin, g/L } \\
\quad \geq 100 \\
<100\end{array}$ & $\begin{array}{l}207(85.5) \\
35(14.5)\end{array}$ & $\begin{array}{l}43(82.7) \\
9(17.3)\end{array}$ & 0.602 & & $\begin{array}{l}303(76.9) \\
91(23.1)\end{array}$ & $\begin{array}{l}117(76.0) \\
37(24.0)\end{array}$ & 0.817 & \\
\hline $\begin{array}{l}\text { Tumor size } \\
\quad \leq 4.75 \\
>4.75\end{array}$ & $\begin{array}{l}183(75.6) \\
59(24.4)\end{array}$ & $\begin{array}{l}33(63.5) \\
19(26.5)\end{array}$ & 0.072 & & $\begin{array}{l}276(70.1) \\
118(29.9)\end{array}$ & $\begin{array}{l}103(66.9) \\
51(33.1)\end{array}$ & 0.470 & \\
\hline
\end{tabular}

(Continued) 
Table 3 (Continued).

\begin{tabular}{|c|c|c|c|c|c|c|c|c|}
\hline \multirow[t]{2}{*}{ Factors } & \multicolumn{4}{|c|}{ Young Patients $(<60 y)$} & \multicolumn{4}{|c|}{ Elderly patients ( $\geq 60 \mathrm{y}$ ) } \\
\hline & $\begin{array}{l}\text { Non-PC } \\
\text { Group } 242 \\
\text { (\%) }\end{array}$ & $\begin{array}{l}\text { PC } \\
\text { Group } \\
52(\%)\end{array}$ & $\begin{array}{l}\text { Univariate } \\
\mathbf{p}\end{array}$ & $\begin{array}{l}\text { Multivariate } \\
\text { p }\end{array}$ & $\begin{array}{l}\text { Non-PC } \\
\text { Group } 394 \\
\text { (\%) }\end{array}$ & $\begin{array}{l}\text { PC } \\
\text { Group } \\
154(\%)\end{array}$ & $\begin{array}{l}\text { Univariate } \\
\mathbf{P}\end{array}$ & $\begin{array}{l}\text { Multivariate } \\
\text { p }\end{array}$ \\
\hline $\begin{array}{l}\text { Histologic type } \\
\text { Signet-ring cell carcinoma } \\
\text { Differentiated } \\
\text { Undifferentiated }\end{array}$ & $\begin{array}{l}40(16.5) \\
199(82.2) \\
3(1.3)\end{array}$ & $\begin{array}{l}7(13.5) \\
45(86.5) \\
0(0.0)\end{array}$ & 0.609 & & $\begin{array}{l}4 I(10.4) \\
34 I(86.5) \\
I 2(3 . I)\end{array}$ & $\begin{array}{l}10(6.5) \\
142(92.2) \\
2(1.3)\end{array}$ & 0.170 & \\
\hline $\begin{array}{l}\text { Pathologic type } \\
\text { Ulcerative type } \\
\text { Non-ulcerative type }\end{array}$ & $\begin{array}{l}193(79.8) \\
49(20.2)\end{array}$ & $\begin{array}{l}48(92.3) \\
4(7.7)\end{array}$ & $0.033^{*}$ & 0.038 & $\begin{array}{l}316(80.2) \\
78(19.8)\end{array}$ & $\begin{array}{l}120(77.9) \\
34(22.1)\end{array}$ & 0.552 & \\
\hline $\begin{array}{l}\text { TNM stage } \\
\text { O/I } \\
\text { II } \\
\text { III }\end{array}$ & $\begin{array}{l}89(36.8) \\
36(14.9) \\
117(48.3)\end{array}$ & $\begin{array}{l}I I(2 \mid .2) \\
7(13.5) \\
34(65.3)\end{array}$ & 0.062 & & $\begin{array}{l}129(32.7) \\
67(17.0) \\
198(50.3)\end{array}$ & $\begin{array}{l}38(24.7) \\
18(11.7) \\
98(63.6)\end{array}$ & $0.018^{*}$ & \\
\hline $\begin{array}{l}\mathrm{N} \text { stage } \\
\text { N0 } \\
\text { NI } \\
\text { N2 } \\
\text { N3 }\end{array}$ & $\begin{array}{l}114(47.1) \\
30(12.4) \\
43(17.8) \\
55(22.7)\end{array}$ & $\begin{array}{l}18(34.6) \\
6(11.5) \\
8(15.4) \\
20(38.5)\end{array}$ & 0.121 & & $\begin{array}{l}176(44.7) \\
51(12.9) \\
65(16.5) \\
102(25.9)\end{array}$ & $\begin{array}{l}54(35.1) \\
24(15.6) \\
32(20.8) \\
44(28.5)\end{array}$ & 0.217 & \\
\hline $\begin{array}{l}\text { T stage } \\
\text { Tis/l } \\
\text { T2 } \\
\text { T3 } \\
\text { T4 }\end{array}$ & $\begin{array}{l}58(24.0) \\
50(20.7) \\
46(19.0) \\
88(36.3)\end{array}$ & $\begin{array}{l}5(9.6) \\
10(19.2) \\
10(19.2) \\
26(50)\end{array}$ & 0.162 & & $\begin{array}{l}83(21.1) \\
91(23.1) \\
79(20.1) \\
141(35.7)\end{array}$ & $\begin{array}{l}19(12.3) \\
32(20.8) \\
30(19.5) \\
73(47.4)\end{array}$ & $0.034^{*}$ & \\
\hline $\begin{array}{l}\text { Tumor location } \\
\text { Cardia } \\
\text { Corpus } \\
\text { Pylorus } \\
\text { Total }\end{array}$ & $\begin{array}{l}29(12.0) \\
55(22.7) \\
157(64.9) \\
I(0.4)\end{array}$ & $\begin{array}{l}\mathrm{II}(2 \mathrm{I} .2) \\
8(15.4) \\
32(6 \mathrm{I} .5) \\
\mathrm{I}(\mathrm{I} .9)\end{array}$ & 0.156 & & $\begin{array}{l}83(21.1) \\
86(21.8) \\
223(56.6) \\
2(0.5)\end{array}$ & $\begin{array}{l}32(20.8) \\
44(28.6) \\
76(49.4) \\
2(1.2)\end{array}$ & 0.246 & \\
\hline $\begin{array}{l}\text { Anastomotic method } \\
\text { Billroth I } \\
\text { Billroth II } \\
\text { Roux-en-y }\end{array}$ & $\begin{array}{l}146(60.3) \\
4 \mid(16.9) \\
55(22.7)\end{array}$ & $\begin{array}{l}26(50) \\
9(17.3) \\
17(32.7)\end{array}$ & 0.284 & & $\begin{array}{l}224(56.9) \\
67(17.0) \\
103(26.1)\end{array}$ & $\begin{array}{l}67(43.5) \\
26(16.9) \\
61(39.6)\end{array}$ & $0.005^{*}$ & \\
\hline $\begin{array}{l}\text { Total gastrectomy } \\
\text { No } \\
\text { Yes }\end{array}$ & $\begin{array}{l}199(82.2) \\
43(17.8)\end{array}$ & $\begin{array}{l}39(75.0) \\
13(25.0)\end{array}$ & 0.228 & & $\begin{array}{l}316(80.2) \\
78(19.8)\end{array}$ & $\begin{array}{l}103(66.9) \\
51(33.1)\end{array}$ & $0.00 I^{*}$ & \\
\hline $\begin{array}{l}\text { Combined operation } \\
\text { No } \\
\text { Yes }\end{array}$ & $\begin{array}{l}226(93.4) \\
16(6.6)\end{array}$ & $\begin{array}{l}47(90.4) \\
5(9.6)\end{array}$ & 0.445 & & $\begin{array}{l}355(85.0) \\
39(15.0)\end{array}$ & $\begin{array}{l}132(85.7) \\
22(14.3)\end{array}$ & 0.142 & \\
\hline $\begin{array}{l}\text { Surgical method } \\
\text { Open } \\
\text { Laparoscopic }\end{array}$ & $\begin{array}{l}212(87.6) \\
30(12.4)\end{array}$ & $\begin{array}{l}5 I(98.1) \\
I(1.9)\end{array}$ & $0.026^{*}$ & & $\begin{array}{l}348(88.3) \\
46(I I .7)\end{array}$ & $\begin{array}{l}\text { I43(92.9) } \\
\text { II (7.I) }\end{array}$ & 0.118 & \\
\hline
\end{tabular}

Note: *Statistically significant $(\mathrm{P}<0.05)$.

Abbreviations: BMI, body mass index; TNM, Tumor-Lymph, Node, Metastasis. 


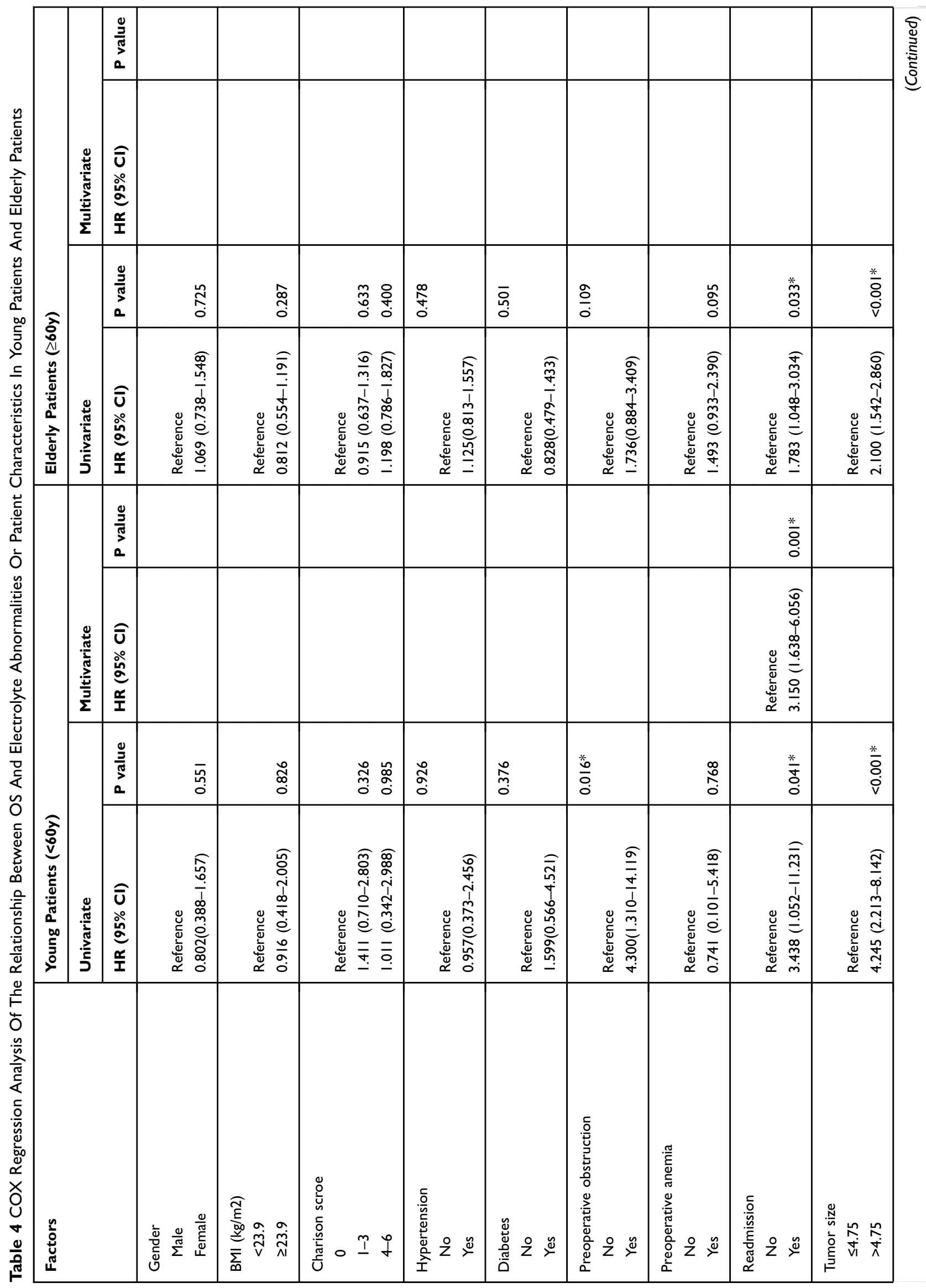




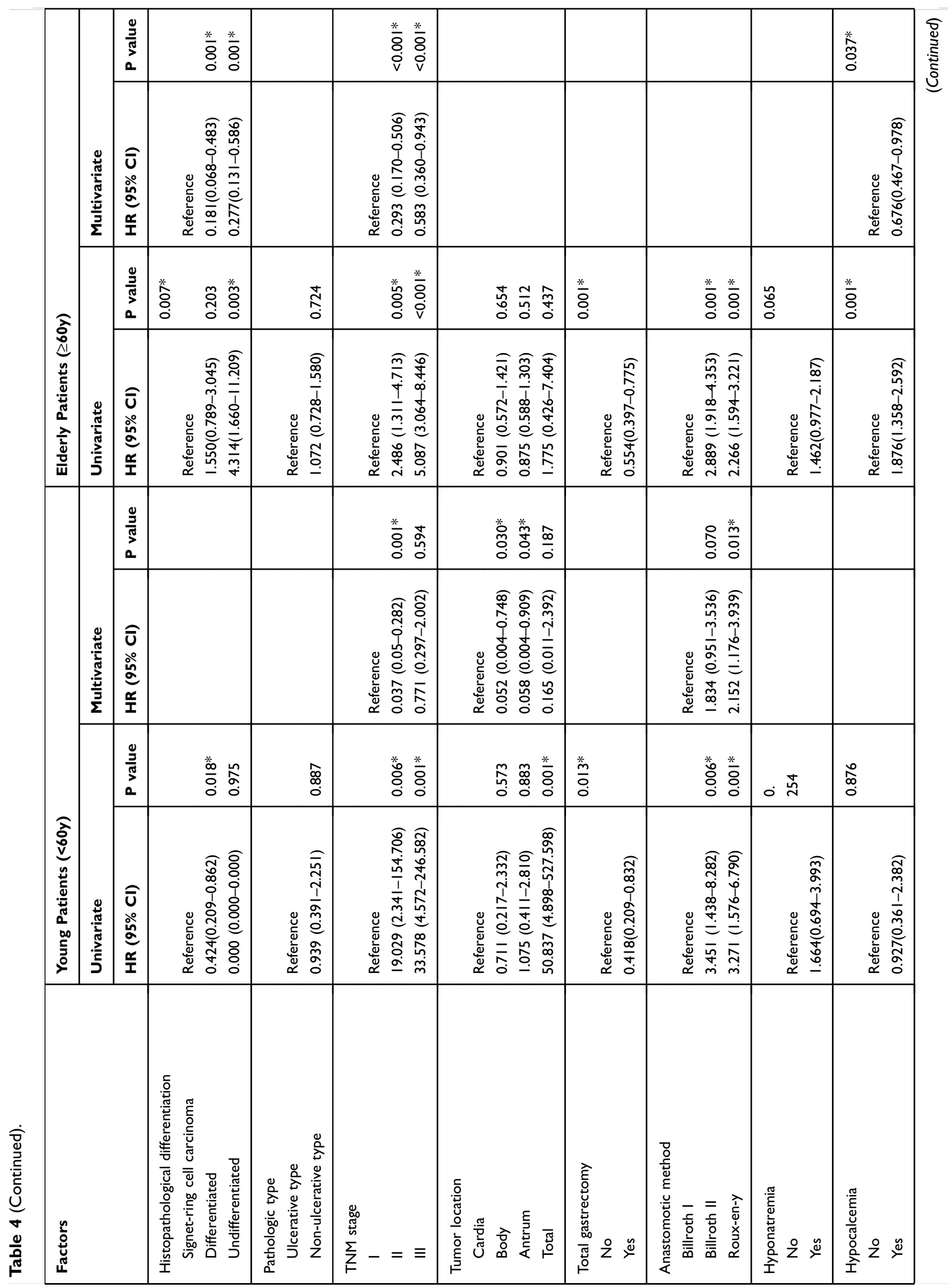




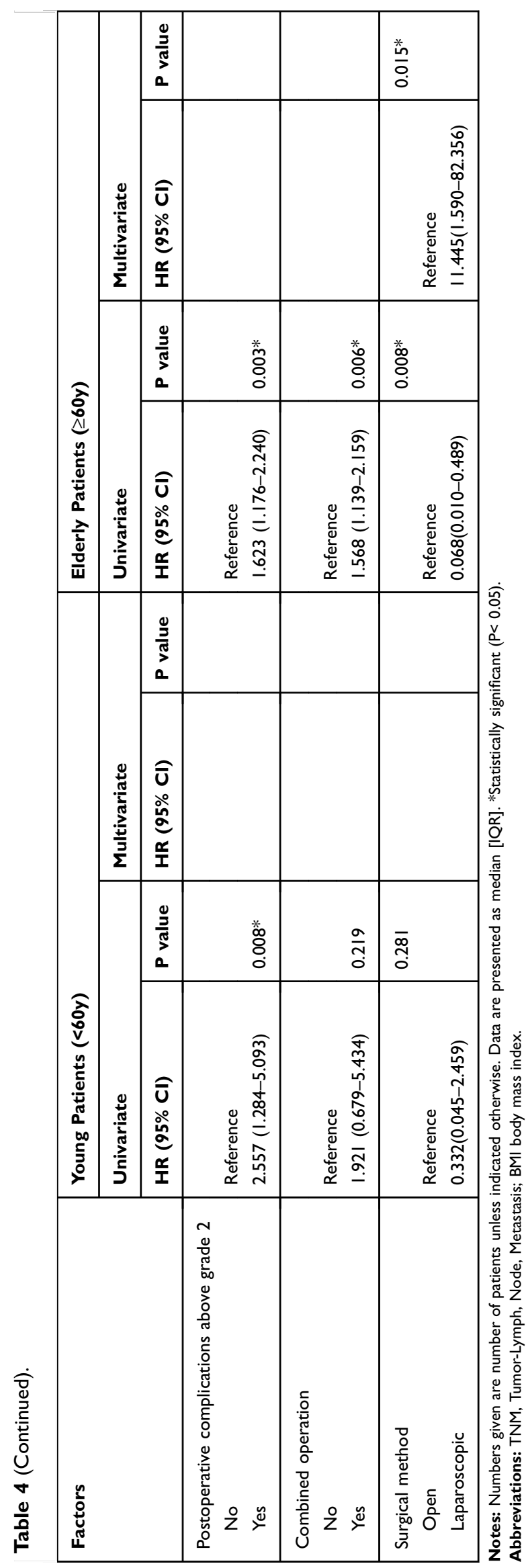

\section{Discussion}

This study retrospectively investigated the impact of preoperative hyponatremia or hypocalcemia on PCs and OS in GC patients by age group. Our results demonstrated that GC patients with hyponatremia or hypocalcemia had a higher risk of developing PCs. PCs not only reduce compliance to succeeding treatment, but also increase medical costs and impair quality of life, and ultimately result in poor prognosis. ${ }^{19,20}$

We also found that the incidence of hyponatremia and hypocalcemia was higher in the elderly group. Considering the physiological differences between young patients and elderly patients, we first examined whether hyponatremia or hypocalcemia was correlated with outcome in the young and the elderly patient groups. Interestingly, hyponatremia or hypocalcemia was an independent risk factor for PCs in the elderly group (hyponatremia, $\mathrm{P}=0.001$; hypocalcemia, $\mathrm{P}=0.003$ ), but it was not significant on univariate analysis in the young group. Moreover, similar to the results of other studies, ${ }^{21-23}$ we found that OS was significantly shorter in elderly GC patients with preoperative hypocalcemia or hyponatremia, and hypocalcemia was found to be an independent risk factor of OS in the elderly group ( $\mathrm{HR}=0.676, \mathrm{P}=0.037)$, but not in the young group. This may be explained by the fact that the relationship between chronic illness and certain factors such as malnutrition and immobility make elderly patients particularly vulnerable to hyponatremia or hypocalcemia. Furthermore, the value of hyponatremia or hypocalcemia as a predictor for PCs and OS in elderly patients may be blunted by age and agerelated poor physical condition. Additionally, considering the same diagnostic criteria and their better fundamental physical condition, young patients with hyponatremia or hypocalcemia may have better nutritional intake than elderly patients with hyponatremia or hypocalcemia. Although preoperative hyponatremia or hypocalcemia is associated with a poorer prognosis in elderly GC patients, there are still questions as to whether hyponatremia or hypocalcemia is a marker of disease severity ${ }^{24}$ and if the correction of hyponatremia or hypocalcemia is beneficial for OS. ${ }^{25,26}$

Cancer outcomes have improved over the last few decades, but the prognostic impact of hyponatremia or hypocalcemia in cancer remains unclear. A study has suggested that correction of hyponatremia or hypocalcemia improves survival, ${ }^{27}$ but the mechanisms behind these relationships are still ambiguous and poorly understood. In 
(A)

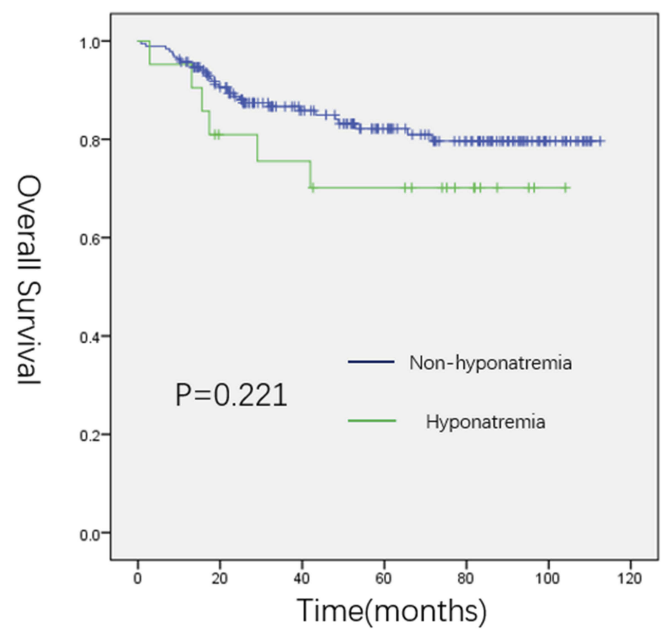

(B)

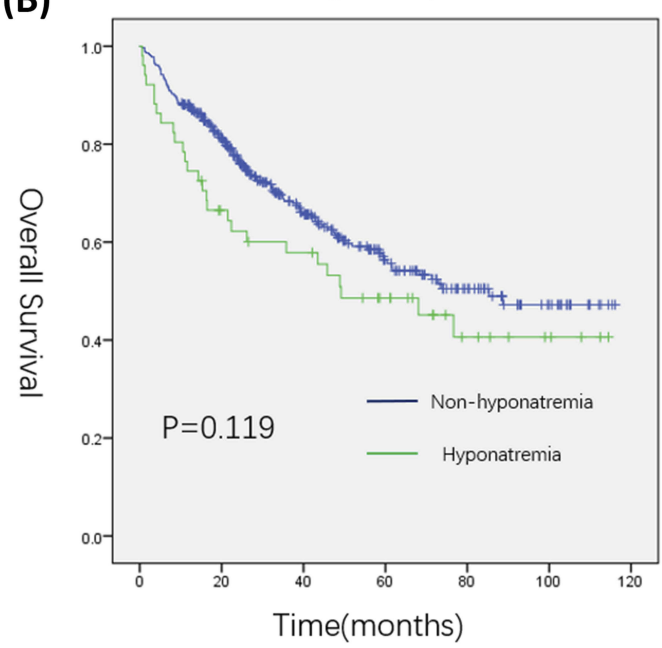

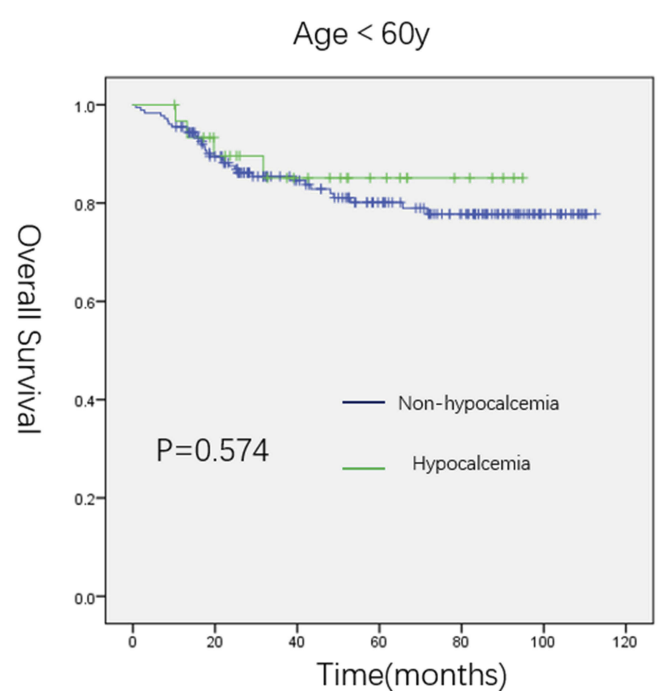

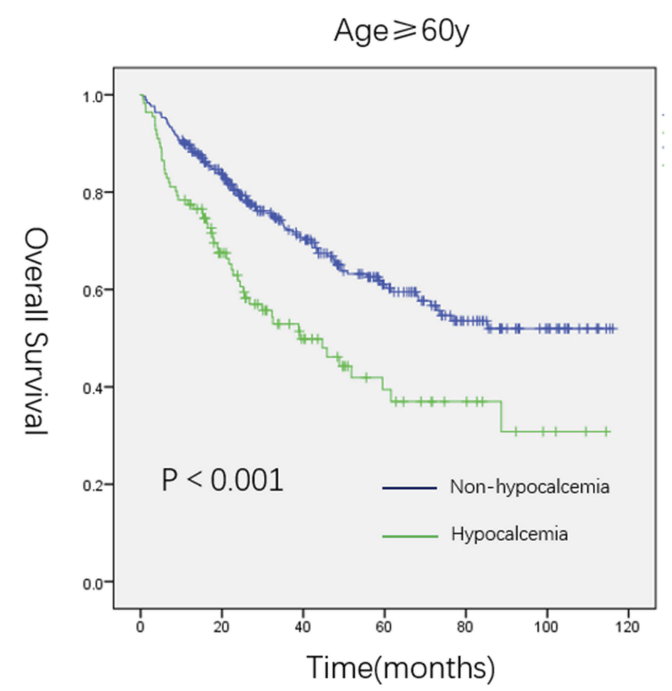

Figure 2 Kaplan-Meier curves. (A) Kaplan-Meier curve for overall survival (OS) in younger patients with hyponatremia or hypocalcemia and those without. (B) KaplanMeier curve for overall survival (OS) in elderly patients with hyponatremia or hypocalcemia and those without.

cancer patients, hyponatremia is usually caused by inadequate antidiuretic hormone (SIADH) secretion by the tumor. $^{28,29}$ In this case, a hyponatremic state is mainly induced by the ectopic secretion of arginine vasopressin from the tumor tissue. ${ }^{30}$ Although SIADH may be the cause of hyponatremia, there is little evidence that SIADH is the primary cause for hyponatremia in GC patients. In the present study, whether hyponatremia was related to SIADH could not be assessed; thus, their potential relationship remains to be further clarified. However, there are some possible causes of hyponatremia in patients with gastric cancer. A subset of chemotherapy drugs can cause hyponatremia by affecting the cytotoxicity of the central nervous system. In fact, chemotherapy or radical gastrectomy is usually accompanied by severe stress reaction, which may lead to or aggravate hyponatremia.
However, according to current research, the actual mechanism of poor PCs and OS due to hyponatremia is still not very clear. Renal or endocrine dysfunction, cancer cells producing antidiuretic hormone $(\mathrm{ADH})$ and higher than normal levels of $\mathrm{ADH}$ in the microenvironment of gastric cancer are reasonable potential explanations.

As for hypocalcemia, one study indicated that impaired renal function and increased renal tubular calcium resorption may cause hypocalcemia. Deterioration of the kidney function itself may be an indication of inflammation. The proinflammatory pathways is mainly regulated by the tumor microenvironment, and this plays an important role in tumor progression. Tumor-induced inflammation may alters endothelial/vascular kidney function, and this leads to reduced calcium reabsorption and diminished renal function. ${ }^{31}$ However, this needs to be verified. 
Although our findings suggest that hyponatremia or hypocalcemia is an adverse prognostic factor, it is unclear if hyponatremia or hypocalcemia is the result of multiple pathophysiological effects or an independent biological factor. Additional research is needed to clarify this.

This is the first study to focus on hyponatremia or hypocalcemia in GC patients, and we evaluated elderly patients who have higher risk of PCs and worse clinical outcome than young patients. Findings from another study suggest that correction of sodium and calcium concentration levels in GC patients with severe hyponatremia or hypocalcemia allows for additional treatments and result in significantly better OS. ${ }^{32}$ Thus, we propose that more attention should be paid to improve the hyponatremic or hypocalcemia status in elderly patients. The urgency and aggressiveness of treatment for elderly patients is predicated on both the serum calcium and serum sodium, and importantly, the manifesting symptoms. Fluid restriction (generally to $500 \mathrm{~mL}$ less than the daily urine output) is a common treatment strategy for transient hyponatremia. However, it may be difficult to perform in elderly GC patients. ${ }^{33}$ Because hydration is an indispensable step in chemotherapy, and fluid-limiting measures can affect nutrient absorption and quality of life. Thus, we recommend a new and physiologically based therapy for elderly GC patients with hyponatremia.

The site of vasopressin action in the distal tubule and may antagonize vasopressin type 2 receptors, leading to water retention. ${ }^{34}$ In addition, given that the treatment of hypocalcemia in elderly GC patients is aimed to improve serum calcium concentration by treating underlying diseases, particularly by reducing urinary excretion of calcium; promoting calcium absorption in the bone; and, to a lesser extent, increasing intestinal calcium absorption, the blood sodium and blood calcium level should be maintained at stable levels before surgery. Meanwhile, although hyponatremia or hypocalcemia also needs to be treated in young patients and blood sodium and calcium levels should also be stabilized, treatment indications can be lightened.

There are several limitations in this study that should not be overlooked. First, due to technical and conditional restrictions, we only chose to investigate hyponatremia and hypocalcemia, and they were not rated according to their severity. Therefore, we will continue to study the postoperative effects of other types of electrolyte disorders on GC patients and rank them by severity. Second, all the data measured in our study were obtained from the first blood collection after admission, and clinicians tend to correct the hyponatremia or hypocalcemia after its diagnosis. Whether this has an impact on the prognosis still needs to be further studied. Third, bias in population selection may be inevitable due to the single-center design. Therefore, a large-scale multicenter trial is necessary to confirm our findings. Fourth, we only analyzed two age categories at a cutoff of 60 years. The effect of hyponatremia or hypocalcemia in different age groups needs to be further studied. Finally, the follow-up period in the present study is less than 5 years, and long-term follow-up data need to be further acquired.

\section{Conclusions}

This is the first retrospective study to identify hyponatremia and hypocalcemia as independent risk factors for PCs and OS in GC patients. Additionally, hyponatremia and hypocalcemia were independent predictors of poor outcomes only in elderly GC patients, and not in young patients. Therefore, greater attention should be paid to hyponatremia or hypocalcemia in elderly GC patients.

\section{Acknowledgments}

This study was funded by National Natural Science Foundation of China (grant no.81602165), the Department of Health of Zhejiang Province, China (grant no. 2016DTA006) and the Wenzhou Municipal Science and Technology Bureau (grant no.Y20150057).

\section{Author Contributions}

All authors contributed to data analysis, drafting or revising the article, gave final approval of the version to be published, and agree to be accountable for all aspects of the work.

\section{Disclosure}

The authors report no conflicts of interest in this work.

\section{References}

1. Bray F, Ferlay J, Soerjomataram I, et al. Global cancer statistics 2018: GLOBOCAN estimates of incidence and mortality worldwide for 36 cancers in 185 countries. CA Cancer J Clin. 2018;68:394-424. doi:10.3322/caac. 21492

2. Nomura S, Sakamoto H, Glenn S, et al. Population health and regional variations of disease burden in Japan, 1990-2015: a systematic subnational analysis for the Global Burden of Disease Study. Lancet. 2015;2017:390.

3. Pan R, Zhu M, Yu C, et al. Cancer incidence and mortality: A cohort study in China, 2008-2013. Int J Cancer. 2017;141:1315-1323. doi:10.1002/ijc.30825 
4. Chen JH, Hsu SN, Huang TC, et al. Prognostic significance of initial serum albumin and $24 \mathrm{hr}$ daily protein excretion before treatment in multiple myeloma. PLoS One. 2015;10:e0128905.

5. Saito Y, Uraoka T, Yamaguchi Y, et al. A prospective, multicenter study of 1111 colorectal endoscopic submucosal dissections (with video). Gastrointest Endosc. 2010;72:1217-1225. doi:10.1016/j.gie.2010.08.004

6. Imamura H, Kurokawa Y, Kawada J, et al. Influence of bursectomy on operative morbidity and mortality after radical gastrectomy for gastric cancer: results of a randomized controlled trial. World J Surg. 2011;35:625-630. doi:10.1007/s00268-010-0914-5

7. Asaka M. A new approach for elimination of gastric cancer deaths in Japan. Int J Cancer. 2013;132:1272-1276. doi:10.1002/ijc.27965

8. Doshi SM, Shah P, Lei X, Lahoti A, Ak S. Hyponatremia in hospitalized cancer patients and its impact on clinical outcomes. Am J Kidney Dis. 2012;59:222-228. doi:10.1053/j.ajkd.2011.08.029

9. Ingles GAH, Ang JE, Ameratunga M, et al. A study of 1088 consecutive cases of electrolyte abnormalities in oncology phase I trials. Eur J Cancer. 2018;104:32-38. doi:10.1016/j.ejca.2018.08.019

10. National Institute of Cancer. Common Terminology criteria foradverse events (CTCAE). NIH Publ. 2010;2009:0e71. doi:10.1080/ 00140139.2010 .489653

11. Kim WR, Biggins SW, Kremers WK, et al. Hyponatremia and mortality among patients on the liver-transplant waiting list. $N$ Engl J Med. 2008;359:1018-1026. doi:10.1056/NEJMoa0801209

12. Pfeffer MA, Claggett B, Assmann SF, et al. Regional variation in patients and outcomes in the Treatment of Preserved Cardiac Function Heart Failure With an Aldosterone Antagonist (TOPCAT) trial. Circulation. 2015;131:34-42. doi:10.1161/CIRCULATIONAHA.114. 013255

13. Waikar SS, Mount DB, Gc C. Mortality after hospitalization with mild, moderate, and severe hyponatremia. Am J Med. 2009;122:857865. doi:10.1016/j.amjmed.2009.01.027

14. Rosner MH, Ac D. Onco-nephrology: the pathophysiology and treatment of malignancy-associated hypercalcemia. Clin J Am Soc Nephrol. 2012;7:1722-1729. doi:10.2215/CJN.02470312

15. Provisional guidelines on standard international age classifications. Statistical papers, series M. New York, US. 1982.

16. Daniel D, Nicolas D, CJAoS P-A. Classification of surgical complications: a new proposal with evaluation in a cohort of 6336 patients and results of a survey. Ann Surg. 2004;240:205-213.

17. Clavien PA, Barkun J, de Oliveira ML, et al. The Clavien-Dindo classification of surgical complications: five-year experience. Ann Surg. 2009;250:187-196. doi:10.1097/SLA.0b013e3181b13ca2

18. Rosner MH, Ac D. Electrolyte disorders associated with cancer. $A d v$ Chronic Kidney Dis. 2014;21:7-17. doi:10.1053/j.ackd.2013.05.005

19. Tokunaga M, Tanizawa Y, Bando E, Kawamura T, Terashima M. Poor survival rate in patients with postoperative intra-abdominal infectious complications following curative gastrectomy for gastric cancer. Ann Surg Oncol. 2013;20:1575-1583. doi:10.1245/s10434012-2720-9
20. Chawla A, Sterns RH, Nigwekar SU, Cappuccio JD. Mortality and serum sodium: do patients die from or with hyponatremia? Clin J Am Soc Nephrol. 2011;6:960-965. doi:10.2215/CJN.10101110

21. Choi JS, Bae EH, Ma SK, Kweon SS, Sw K. Prognostic impact of hyponatraemia in patients with colorectal cancer. Colorectal Dis. 2015;17:409-416. doi:10.1111/codi.12878

22. Clines GA, Ta G. Hypercalcaemia of malignancy and basic research on mechanisms responsible for osteolytic and osteoblastic metastasis to bone. Endocr Relat Cancer. 2005;12:549-583. doi:10.1677/ erc. 1.00543

23. Balachandran K, Okines A, Gunapala R, Morganstein D, Popat S. Resolution of severe hyponatraemia is associated with improved survival in patients with cancer. BMC Cancer. 2015;15:163. doi:10.1186/ s12885-015-1156-6

24. Schrier RW, Sharma S, Shchekochikhin D. Hyponatraemia: more than just a marker of disease severity? Nat Rev Nephrol. 2013;9:37-50. doi:10.1038/nrneph.2012.246

25. Castillo JJ, Glezerman IG, Boklage SH, et al. The occurrence of hyponatremia and its importance as a prognostic factor in a crosssection of cancer patients. BMC Cancer. 2016;16:564. doi:10.1186/ s12885-016-2610-9

26. Castillo JJ, Vincent M, Justice E. Diagnosis and management of hyponatremia in cancer patients. Oncologist. 2012;17:756-765. doi:10.1634/theoncologist.2011-0400

27. Abu Zeinah GF, Al-Kindi SG, Hassan AA, Allam A. Hyponatraemia in cancer: association with type of cancer and mortality. Eur J Cancer Care (Engl). 2015;24:224-231. doi:10.1111/ecc.12187

28. Tiseo M, Buti S, Boni L, Mattioni R, Ardizzoni A. Prognostic role of hyponatremia in 564 small cell lung cancer patients treated with topotecan. Lung Cancer. 2014;86:91-95. doi:10.1016/j.lungcan.2014. 07.022

29. Onitilo AA, Kio E, Doi SA. Tumor-related hyponatremia. Clin Med Res. 2007;5:228-237. doi:10.3121/cmr.2007.762

30. Coulson R, Seng HL, Connelly AA, et al. The Angiotensin Receptor Blocker, Losartan, Inhibits Mammary Tumor Development and Progression to Invasive Carcinoma. Vol. 8. 2017:18640.

31. Fried LF, Katz R, Sarnak MJ, et al. Kidney function as a predictor of noncardiovascular mortality. J Am Soc Nephrol. 2005;16:3728-3735.

32. Glezerman IG, Sternlicht H. Hypercalcemia of malignancy and new treatment options. Ther Clin Risk Manag. 2015. doi:10.2147/tcrm. S83681

33. Morizane S, Yumioka T, Yamaguchi N, et al. Risk stratification model, including preoperative serum $\mathrm{C}$-reactive protein and estimated glomerular filtration rate levels, in patients with upper urinary tract urothelial carcinoma undergoing radical nephroureterectomy. Int Urol Nephrol. 2015;47:1335-1341.

34. Panotopoulos J, Posch F, Funovics PT, et al. Elevated serum creatinine and low albumin are associated with poor outcomes in patients with liposarcoma. J Orthop Res. 2016;34:533-538.

\section{Publish your work in this journal}

Cancer Management and Research is an international, peer-reviewed open access journal focusing on cancer research and the optimal use of preventative and integrated treatment interventions to achieve improved outcomes, enhanced survival and quality of life for the cancer patient.
The manuscript management system is completely online and includes a very quick and fair peer-review system, which is all easy to use. Visit http://www.dovepress.com/testimonials.php to read real quotes from published authors. 